\title{
The RCW 114 nebula: An old supernova remnant or a WR wind-blown bubble?
}

\author{
B. Y. Welsh ${ }^{1}$, S. Sallmen ${ }^{1}$, S. Jelinsky ${ }^{1}$, and R. Lallement ${ }^{2}$ \\ ${ }^{1}$ Experimental Astrophysics Group, Space Sciences Laboratory, UC Berkeley, Berkeley, CA 94720, USA \\ 2 Service d'Aéronomie du CNRS, 91371 Verrières-le-Buisson, France \\ Received 25 October 2002 / Accepted 4 February 2003

\begin{abstract}
We present medium resolution spectra $\left(R \sim 7 \mathrm{~km} \mathrm{~s}^{-1}\right)$ of the interstellar NaI D1 \& D2 absorption lines observed towards 7 early-type stars with distances ranging from 221 to $\sim 1.5 \mathrm{kpc}$ in the line-of-sight to the RCW 114 nebula. Our observed pattern of absorption is in conflict with the findings of Bedford et al. (1984) who placed an upper limit to the distance to this nebula of $<200 \mathrm{pc}$. Instead, our spectral data are consistent with a marked increase in NaI absorption complexity occuring only for sight-lines with distances $>1 \mathrm{kpc}$. A similar pattern of absorption behavior is seen for the SiII $\lambda 1304 \AA$ interstellar line observed in the ultraviolet towards four of the aforementioned stars. We propose a scenario in which the absorption components with velocities $<-10 \mathrm{~km} \mathrm{~s}^{-1}$ seen towards RCW 114 are caused by an expanding stellar wind-blown bubble associated with the Wolf-Rayet (WR) star, HD $156385(d \sim 1.5 \mathrm{kpc})$, and that the $\mathrm{H} \alpha$ and [SII] nebular emission probably arises in shocked filamentary gas associated with a pre-existing evolved supernova remnant cavity produced by the high-mass progenitor of the present WR star.
\end{abstract}

Key words. ISM: bubbles - ISM: supernova remnants

\section{Introduction}

$\operatorname{RCW} 114\left(l=343.9^{\circ}, b=-4.8^{\circ}\right)$ is a large, highly filamentary gas nebula extending over an angular diameter of $\sim 4^{\circ}$ on the sky. Based on its optical $\mathrm{H} \alpha+[\mathrm{NII}]$ appearance, Meaburn et al. (1977) first tentatively suggested that this object was probably an old supernova remnant (SNR). Further $\mathrm{H} \alpha$ and [SII] mappings of the ionized gas in RCW 114 by Meaburn et al. (1991) revealed a global nebular expansion velocity of $25-35 \mathrm{~km} \mathrm{~s}^{-1}$, such that the derived kinetic energy of the expanding shell (based on IRAS far infrared flux densities) gave a value consistent with that expected from a Type II supernova event. Recent multifibre visible spectroscopy ( $\lambda 3926 \AA-7038 \AA$ ) of the gas filaments of RCW 114 have revealed a pattern of optical emission with $[\mathrm{SII}] / \mathrm{H} \alpha$ line intensity ratios consistent with their production by an evolved remnant that is interacting with the ambient interstellar medium (Walker \& Zealey 2001).

Bedford et al. (1984), hereafter (B84), recorded high resolution absorption spectra of the interstellar NaI D2 line (5890 ̊) towards 7 stars in line-of-sight towards RCW 114 and detected an inconsistent pattern of absorption features spanning the velocity range +5 to $-24 \mathrm{~km} \mathrm{~s}^{-1}$ that they tentatively attributed to the disruption of the ambient interstellar gas by an old supernova event. Based on the photometric distances derived for the stars that exhibited these nebular absorption components, they derived a distance to RCW 114 of $<200$ pc.

Send offprint requests to: $\mathrm{B}$. Y. Welsh,

e-mail: bwelsh@ssl. berkeley.edu
This would make RCW 114 the closest known SNR to the Sun, and from this distance a diameter of $<17.5 \mathrm{pc}$ and an age of 20000 years could be derived for the remnant.

The placement of a SNR so close to the Sun might possibly explain the formation of the Local Bubble (LB), which is a hot $\left(T \sim 10^{6} \mathrm{~K}\right)$ and rarefied $\left(n_{\mathrm{H}} \sim 0.005 \mathrm{~cm}^{-3}\right)$ region of space approximately $150 \mathrm{pc}$ in diameter that surrounds the Sun (Frisch 1995). Recently we have completed a preliminary mapping of the neutral gas boundary to the LB using the interstellar NaI D-lines observed in absorption towards some 456 stellar targets lying within 250 pc (Sfeir et al. 1999). From these data we have been able to derive the approximate shape of the hot LB cavity in the galactic plane and the pattern of these absorption data were inconsistent with the presence of such a nearby SNR. Our interest in RCW 114 was further intensified since we are currently carrying out a research program to identify suitable background stellar targets that can be used (with FUSE and HST-STIS observations) to probe the disturbed gas in nearby $(d<2 \mathrm{kpc})$ SNRs (Welsh et al. 2002; Sfeir 1999). Since the original work of B84 did not have access to the recent Hipparcos satellite data set of stellar distances (ESA 1997), we decided to re-look at their data with respect to the new (more accurate) distances to the stellar targets allegedly responsible for the absorption components associated with the expansion of the RCW 114 SNR shell.

Rather surprisingly we found that HD $157042(d=221 \mathrm{pc})$ - a star that B84 had identified as possessing absorption components associated with the SNR shell expansion - appeared 
Table 1. Stellar target information and NaI equivalent width measurement.

\begin{tabular}{cccccccc}
\hline \hline Star & $(l, b)$ & $m_{v}$ & Sp & $E(B-V)$ & $\begin{array}{c}\text { distance } \\
(\mathrm{pc})\end{array}$ & $\begin{array}{c}\text { NaI D-2 } \\
(\mathrm{mA})\end{array}$ & $\begin{array}{c}\text { NaI D-1 } \\
(\mathrm{mA})\end{array}$ \\
\hline HD 156385 & $\left(343.2^{\circ},-4.8^{\circ}\right)$ & 6.9 & WC7 & 0.38 & $1550(+500,-400)$ & $625 \pm 25$ & $520 \pm 20$ \\
HD 156575 & $\left(342.9^{\circ},-5.2^{\circ}\right)$ & 7.4 & B0.5III & 0.46 & $1100(+150,-200)$ & $560 \pm 25$ & $500 \pm 20$ \\
HD 157042 & $\left(342.0^{\circ},-6.3^{\circ}\right)$ & 5.2 & B2.5IVe & 0.09 & $221^{*}(+48,-33)$ & $185 \pm 15$ & $115 \pm 10$ \\
HD 157243 & $\left(344.9^{\circ},-4.6^{\circ}\right)$ & 5.1 & B7III & 0.06 & $232^{*}(+50,-35)$ & $165 \pm 15$ & $120 \pm 10$ \\
HD 157698 & $\left(342.7^{\circ},-6.7^{\circ}\right)$ & 7.2 & B7III & 0.11 & $763^{*}(+2100,-220)$ & $255 \pm 25$ & $190 \pm 20$ \\
HD 157832 & $\left(342.8^{\circ},-6.7^{\circ}\right)$ & 6.7 & B2Ve & 0.25 & $780( \pm 350)$ & $335 \pm 20$ & $220 \pm 15$ \\
HD 158042 & $\left(345.5^{\circ},-5.2^{\circ}\right)$ & 6.3 & B5III & 0.10 & $465^{*}(+385,-150)$ & $205 \pm 20$ & $155 \pm 15$ \\
\hline
\end{tabular}

* Hipparcos distance.

to possess stronger and more complex absorption features than stellar targets with far greater (Hipparcos) distances and similar sight-lines through the RCW 114 nebula. Although it is well documented that the neutral absorption structure of SNRs is spatially highly variable (Jenkins et al. 1984), we decided that a further investigation of the RCW 114 line-of-sight was necessary to verify this point. We also noted that HD 157042 is of spectral type B2IIIe and it is now well documented that many Be stars are surrounded by gas and dust disks which can give rise to variable, narrow circumstellar-shell absorption features in their NaI spectra (Hanuschik et al. 1996). Furthermore, we noted that the distribution of interstellar $\mathrm{HI} 21 \mathrm{~cm}$ line emission from the RCW 114 nebula by Cappa de Nicolau et al. (1988) was interpreted as the result of the interaction between the stellar wind of the Wolf-Rayet (WR) star HD 156385 and/or its massive progenitor with the surrounding interstellar medium. However, this scenario was discounted by both Walker \& Zealey (2001) and B84 in favor of a SN origin on the grounds that HD 156385 was thought to lie well beyond the RCW 114 nebula at a distance of $\sim 2 \mathrm{kpc}$.

In this Paper we present high $S / N(>70: 1)$, medium spectral resolution $\left(R \sim 7 \mathrm{~km} \mathrm{~s}^{-1}\right)$ observations of the interstellar NaI D1 and D2 lines detected towards 7 early-type stars with distances ranging from $221 \mathrm{pc}$ to $\sim 1.5 \mathrm{kpc}$ whose absorption sight-lines all pass through the apparent extent of the RCW 114 gas nebula as defined by its $\mathrm{H} \alpha$ emission contours. By investigating the resulting pattern of absorption as a function of distance we have been able to determine that the nominal distance to the RCW 114 nebula must be $>1 \mathrm{kpc}$, well in excess of the value of $<200$ pc suggested by B84. This pattern of absorption is also mimicked by the SiII $\lambda 1304 \AA$ ultraviolet interstellar line observed towards four of the target stars. We propose that the most probable cause of the observed complexity of the $\mathrm{NaI}$ (and SiII) absorption components detected at distances $>1 \mathrm{kpc}$ towards this nebula is the presence of both the stellar winds from the WR star, HD 156385, and an evolved SNR produced $\sim 10^{6}$ years ago by its high-mass progenitor.

\section{Observations}

In Table 1 we list the seven stars we have observed, together with their visual magnitudes, spectral types, reddening values and distance estimates. The positions of these targets with respect to the $\mathrm{H} \alpha$ emission contours of the RCW 114 nebula (as recorded by the Southern $\mathrm{H} \alpha$ Sky Survey Atlas, Gaustad et al. 2001) are shown in Fig. 1. Hipparcos satellite distances are available for 4 of the stars and are marked by an asterisk (*) in Table 1 (ESA 1997). We have estimated the stellar photometric distance (and its associated error) to HD 156575 using the absolute magnitude classifications of Balona \& Crampton (1974) and the reddening value listed by Savage et al. (1985). The distance and reddening estimate for HD 157832 has been taken from Kozok (1985), and similarly such values for the WR star, HD 156385, have been taken from the recent work of Dessart et al. (2000). Our derived distance estimate of $1.55 \pm 0.5 \mathrm{kpc}$ to this WR star is consistent with the marginal Hipparcos parallax measurement of $-0.86 \pm 0.89$ mas. We note that two of our target stars (HD 157042 and HD 157243) were also observed in the NaI D2-line by B84 and were specifically selected for data comparison purposes.

Observations of both interstellar NaI D1 \& D2 lines at $\sim 5890 \AA$ were obtained during the night of May 26th, 2002 using the GIRAFFE fiber-fed echelle spectrograph at the $1.9 \mathrm{~m}$ Radcliffe telescope of the South African Astronomical Observatory. The photon data were recorded on a Tektronix $1024 \times 1024$ CCD detector and the calibrated spectra extracted using on-line procedures written by Luis Balona (see http://www . saao.ac.za/facilities/giraffe/index. htm). Briefly, these procedures consist of background subtraction, flatfielding, optimal spectral order extraction and wavelength assignment from Th-Ar calibration spectra. The resolution of the resultant spectra was $7 \mathrm{~km} \mathrm{~s}^{-1}$ and the wavelength accuracy of the calibrated data was $\sim \pm 0.06 \AA$. All the spectra were well-exposed with typical $S / N$ ratios in excess of 70:1 and all velocities are reported in the heliocentric frame of reference. An absorption spectrum of the bright and nearby B star $\alpha$ Gru (which has no known detectable interstellar NaI) was also recorded to assist in the initial removal process of the narrow telluric water vapor absorption lines that particularly afffect contamination of the NaI D line-profiles. The residual levels of telluric contamination (which for this night's observations were of a very low level) were then removed using a computed synthetic atmospheric transmission spectrum described in Lallement et al. (1993). 


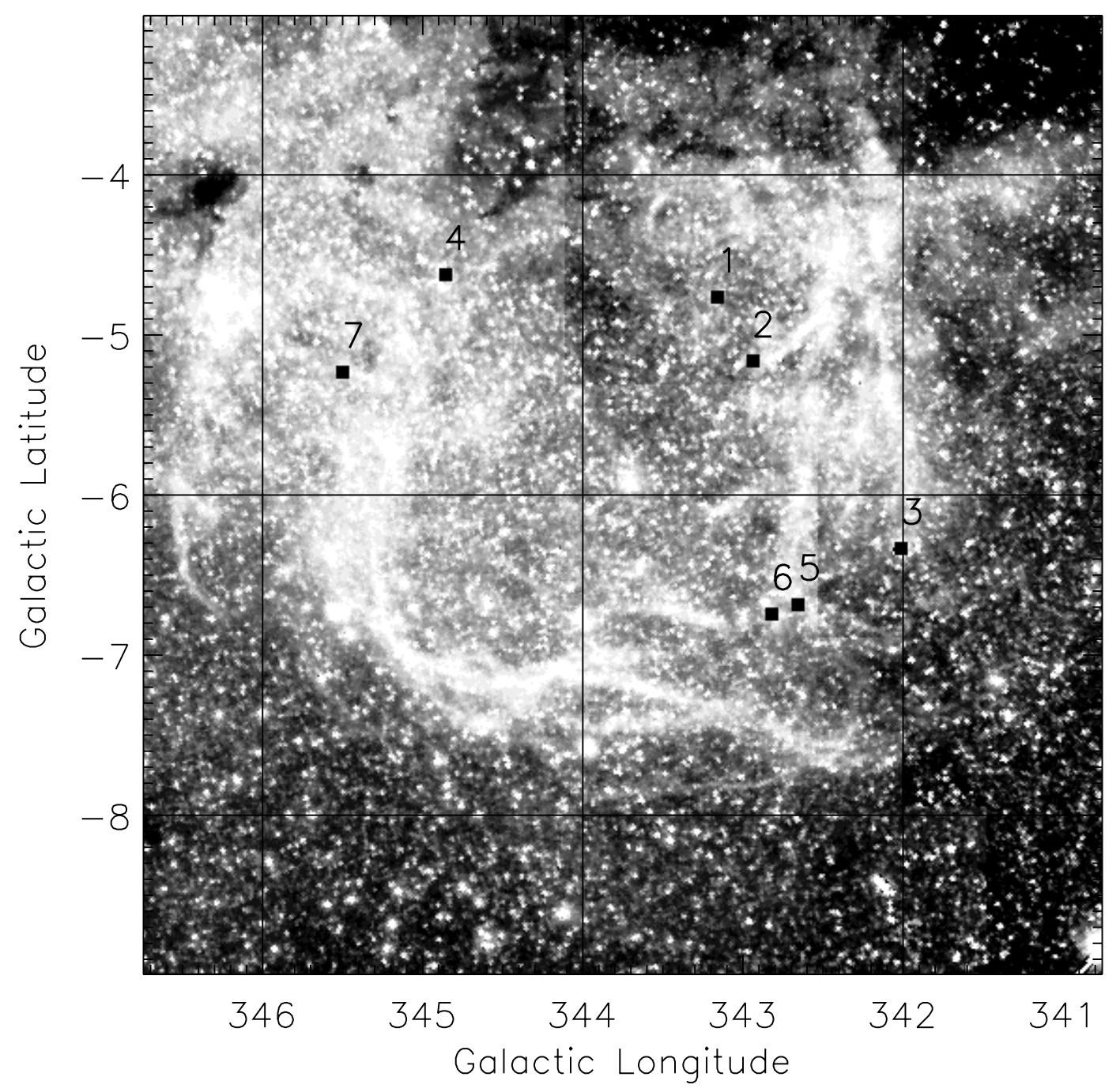

Fig. 1. The RCW 114 nebula as recorded by the Southern H $\alpha$ Sky Survey Atlas (Guastad et al. 2001) showing the positions of the target stars (1) HD 156385, (2) HD 156575, (3) HD 157042, (4) HD 157243, (5) HD 157698, (6) 157832 and (7) HD 158042.

\section{Interstellar analysis}

We have determined the local stellar continua for all of the $\mathrm{NaI}$ D-lines using a multi-order polynomial in order to produce the resultant residual intensity profiles shown in Fig. 2. We show these profiles in the order of increasing sight-line distance. These profiles were then fit with one or more absorption components (identified with interstellar "clouds") using a linefitting program described in Sfeir et al. (1999). This program assigns a 3-parameter theoretical fit to the observed profiles by assigning values for the interstellar gas cloud velocity, $V$, a Gaussian velocity dispersion, $b$ and a cloud component column density, $N$. The best-fit values of $V, b$ and $N$ for the NaI lines towards all 7 targets are listed in Table 2, and the model fits are shown in Fig. 2. The equivalent widths of the interstellar D1 and D2 lines are listed in Table 1, together with estimates of their measurement errors.

It is immediately apparent from Fig. 2 that the NaI spectra recorded towards the 4 sight-lines with distances $<763$ pc can all be fit with a single absorption component centered close to $V \sim-2.5 \mathrm{~km} \mathrm{~s}^{-1}$. These absorption profiles exhibit no obvious asymmetry at the spectral resolution of the data, and thus it would appear that we have failed to detect any significant velocity structure for the four nearest sight-lines. For the remaining 3 sight-lines with distance estimates $>763$ pc the NaI profiles contain a greater complexity of absorption features, with the most distant target (the WR star, HD 156385) requiring a minimum of 4 velocity components to fit its D2 and D1 absorption profiles.

\section{Discussion}

\subsection{Comparison with the results of Beford et al. (1984)}

The present observations of the profiles of both the D1 and D2 lines seen towards the two stars (HD 157042 and HD 157243) that were observed in common with B84 are clearly in disagreement with the pattern of NaI D2 absorptionline features presented by them. We note that our present spectra are of a $S / N$ ratio $>70: 1$, whereas the B84 data have a low 
Table 2. NaI absorption line best-fit parameters (stars listed by increasing distance).

\begin{tabular}{|c|c|c|c|c|c|c|c|c|c|c|c|c|}
\hline Star & $\begin{array}{c}V_{1} \\
\mathrm{~km} \mathrm{~s}^{-1}\end{array}$ & $b_{1}$ & $\begin{array}{c}N_{1} \\
\left(10^{11} \mathrm{~cm}^{-2}\right)\end{array}$ & $\begin{array}{c}V_{2} \\
\mathrm{~km} \mathrm{~s}^{-1}\end{array}$ & $b_{2}$ & $\begin{array}{c}N_{2} \\
\left(10^{11} \mathrm{~cm}^{-2}\right)\end{array}$ & $\begin{array}{c}V_{3} \\
\mathrm{~km} \mathrm{~s}^{-1}\end{array}$ & $b_{3}$ & $\begin{array}{c}N_{3} \\
\left(10^{11} \mathrm{~cm}^{-2}\right)\end{array}$ & $\begin{array}{c}V_{4} \\
\mathrm{~km} \mathrm{~s}^{-1}\end{array}$ & $b_{4}$ & $\begin{array}{c}N_{4} \\
\left(10^{11} \mathrm{~cm}^{-2}\right)\end{array}$ \\
\hline HD 157042 & & & & & & & -3.9 & 3.3 & $13.5 \pm 1.5$ & & & \\
\hline HD 157243 & & & & & & & -1.7 & 0.9 & $24.7 \pm 3.2$ & & & \\
\hline HD 158042 & & & & & & & -1.8 & 1.3 & $26.1 \pm 5.8$ & & & \\
\hline HD 157698 & & & & & & & -3.1 & 3.3 & $25.9 \pm 3.2$ & & & \\
\hline HD 157832 & & & & -9.6 & 1.7 & $21.3 \pm 8.6$ & +0.8 & 2.3 & $13.2 \pm 2.1$ & & & \\
\hline HD 156575 & & & & -13.0 & 1.2 & $200.0 \pm 15$ & -1.4 & 2.8 & $105.5 \pm 15$ & & & \\
\hline HD 156385 & -45.4 & 1.5 & $0.75 \pm 0.2$ & -17.5 & 1.7 & $22.8 \pm 7.2$ & -3.5 & 3.3 & $87.5 \pm 10$ & +3.3 & 0.9 & $105 \pm 15$ \\
\hline
\end{tabular}

$S / N$ ratio $<10: 1$. We believe the main reasons for the differences between the two data sets (and the resultant interpretation of these profiles) can be explained by the following three points:

(i) Although reference is made to the use of strong telluric lines as a wavelength check in the data reduction process performed by B84, unfortunately we have discovered that such lines were not removed from any of their published interstellar D2-line profiles (K. Elliott, private communication). The very strongest telluric features in this wavelength region are given in Fig. 6 of Lallement et al. (1993), which shows strong features due to both telluric water vapor and atmospheric NaI absorption lying within $\sim 25 \mathrm{~km} \mathrm{~s}^{-1}$ of the rest wavelength of NaI. Figure 1 of B84 shows a weak absorption feature appearing at a velocity of $V=-24 \mathrm{~km} \mathrm{~s}^{-1}$ in the spectra of five of the targets that they observed. The authors identified this absorption feature as being of nebular origin in the case of HD 157042. We have not detected this feature in either of the D1 or D2-line spectra recorded towards HD 157052 and thus, taken together with the far superior $S / N$ of our present data, we believe this line component to be of telluric origin.

(ii) The star HD 157042 is a Be star (B2IIIe) and, as commented earlier, has known variable absorption characteristics due to the presence of strong stellar winds and an ambient disk of circumstellar gas. Absorption variability for this star has been observed in the visible FeII and $\mathrm{H} \alpha$ lines by Hanuschik et al. (1996), and narrow variable absorption components have aslo been observed in the ultraviolet stellar lines of CIV by Prinja (1989). Thus, the absorption feature seen in the NaI D2 profile of B84 at $-8.4 \mathrm{~km} \mathrm{~s}^{-1}$, which is not present in either of the D2 or D1 lines in our spectrum of HD 157042, could be plausibly explained by a local variation in circumstellar absorption, and is thus most probably not associated with absorption by the RCW 114 nebular gas.

(iii) Our present observations were taken with a spectral resolution of $7 \mathrm{~km} \mathrm{~s}^{-1}$ compared to that of $\sim 3 \mathrm{~km} \mathrm{~s}^{-1}$ by B84. Our D1 and D2 profiles of HD 157243 shown in Fig. 2 clearly have only one resolved velocity component at $V=$ $-1.7 \mathrm{~km} \mathrm{~s}^{-1}$, wheareas the higher resolution data of $\mathrm{B} 84 \mathrm{re}-$ veal two narrow, closely spaced components with velocities of +4.1 and $-4.1 \mathrm{~km} \mathrm{~s}^{-1}$. Our present profiles, however, show no absorption line-asymmetry and no obvious signs of these two components. Our spectral observations are certainly capable of (partially) resolving components with this velocity separation (i.e. $\sim 8 \mathrm{~km} \mathrm{~s}^{-1}$ ), as demonstrated in the profile of HD 157832 shown in our Fig. 2. Our inability to reproduce the NaI D2 spectra of B84 is thus perplexing, but this discrepancy may also be due to telluric line contamination of their spectra.

\subsection{Is RCW 114 an old SNR or a WR stellar wind-blown bubble?}

The association of the RCW 114 nebula with that of an evolved SNR has historically been based on two main factors; (1) the filamentary appearance of its optical nebulosity revealed by H $\alpha$ imagery (Meaburn \& Rovithis 1977; Meaburn et al. 1991) which strongly resembles that of other galactic SNRs in their momentum conserving phase, and (2) the ratio of the intensities of the [SII] and $\mathrm{H} \alpha$ emission lines observed throughout $~ 50 \%$ of the nebula bracket the range 0.2 to 1.17 , which suggests that such emission is produced by shock excitation (Walker \& Zealey 2001). However, the absence of appreciable levels of radio-continuum and X-ray emission from RCW 114 (which are normally observed towards SNRs) does not support the former interpretation as a SNR.

Alternately, both Heckathorn et al. (1982) and Cappa de Nicolau et al. (1988) have associated the RCW 114 nebula with that of an expanding HI shell of gas driven by the strong stellar-wind from the WR star, HD 156385 (WR 90) and/or its massive progenitor. Neutral bubbles of hydrogen gas have been detected around several WR stars, but the association of the RCW 114 nebula with the WR star HD 156385 has generally been dismissed due to distance arguments. Such arguments rely entirely on the results of B84 who placed the distance of $\mathrm{NaI}$ absorption features that were thought to be associated with the expansion of the RCW 114 nebular gas at $<200 \mathrm{pc}$. In addition that paper quotes a distance to HD 156385 of $\sim 2 \mathrm{kpc}$, which was used as a strong argument against any physical association between the WR star and the allegedly foreground RCW 114 nebula.

A major tenet of the B84 paper is that the additional observed complexity of absorption features seen in the NaI D2-line profiles is only present in the spectra of stars with distances $>200$ pc. Their Fig. 1 presents seven 
HD157042
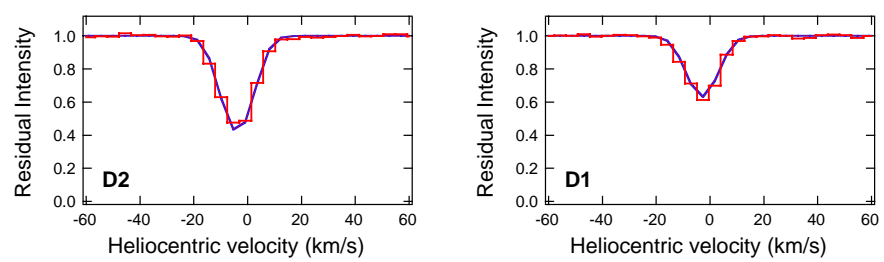

\section{HD157243}
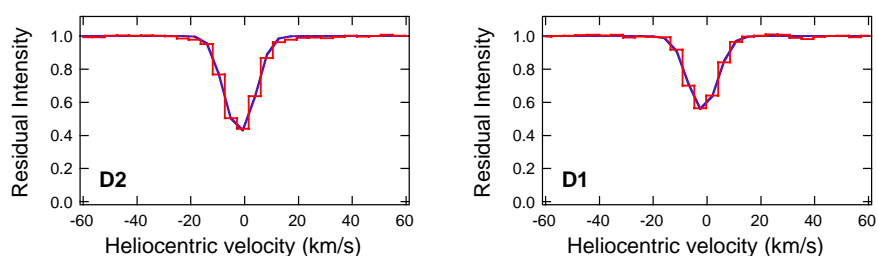

\section{HD158042}
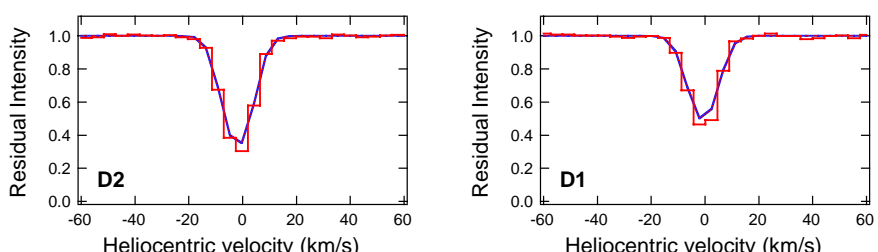

\section{HD157698}
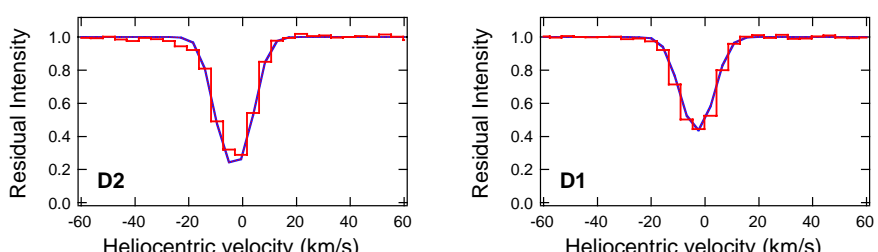

\section{HD157832}
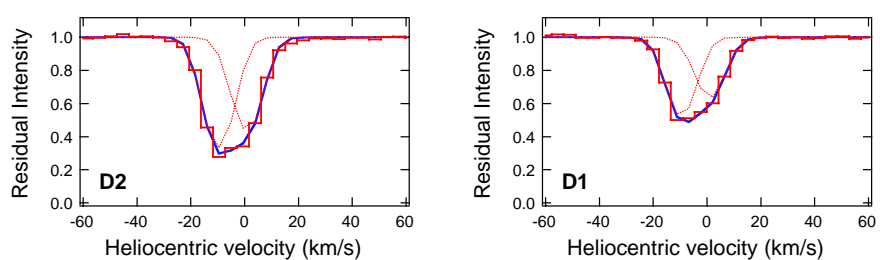

\section{HD156575}
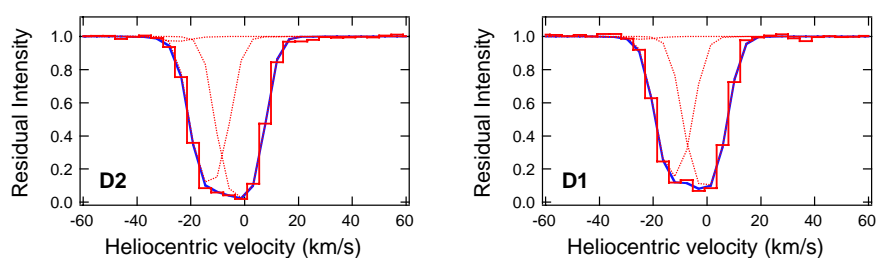

\section{HD156385}
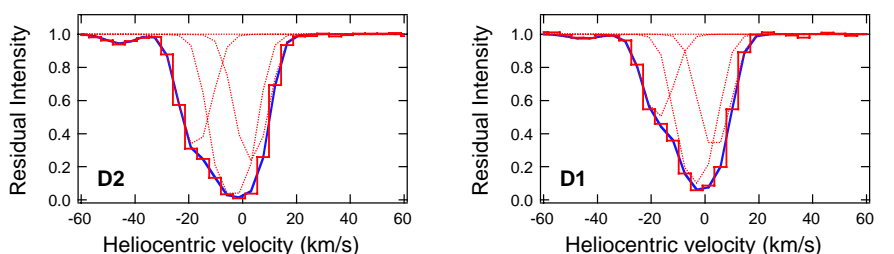

Fig. 2. Interstellar NaI D1 \& D2 absorption line profiles for the seven targets observed towards the RCW 114 nebula. Solid thick lines represent the best-fit model to the observed data points, which are shown by the lighter lines. Actual model absorption components (unconvolved) are shown by the dotted lines. The absorption profiles are presented by increasing distance of the target.
NaI D2-line spectra in order of increasing stellar distance (180 pc to $>1 \mathrm{kpc}$ ), with the simplest absorption profile being associated with the nearest stellar sight-line and more distant sight-lines possessing extra absorption components allegedly due to the interaction of the expanding nebula with the ambient ISM. However, the Hipparcos distances for 4 of their 5 nearest targets are, in fact, essentially the same (i.e. 212-232 pc), and thus their "increasing distance-increasing absorption" explanantion for the origin of the higher-velocity absorption components for the majority of their targets cannot now be sustained in the light of these new and more accurate distance estimates and the possible telluric line contamination of their spectra.

The NaI interstellar lines sample cold and neutral gas and have been widely used as probes of the general interstellar medium (Hobbs 1978). More recently they have been used with great success in the determination of distances to molecular clouds, the neutral boundary to the Local Bubble and the distance to SNRs (Penprase 1993; Sfeir et al. 1999; Welsh et al. 2002). In general, the accuracy of such distance determinations relies both on the knowledge of the stellar distances used to probe the sight-lines and the sampling distance between each stellar target along that sight-line. Our present set of 7 spectra sample the RCW 114 sight-line at distances between 221 pc and $\sim 1.5 \mathrm{kpc}$ (at a sampling frequency of $\sim 200 \mathrm{pc}$ ), and as can be seen in Fig. 3 the NaI absorption equivalent widths approximately double in strength over the path length extending from 220 to $780 \mathrm{pc}$. For the two sight-lines sampled with distances $>1$ kpc (i.e. HD 156575 and HD 156385) the NaI absorption profiles show a very marked increase in absorption equivalent width that is accompanied by an associated increase in interstellar reddening, suggesting that these two sight-lines have encountered a dense complex of interstellar gas.

In Fig. 4 we show the interstellar profiles for the SiII $\lambda 1304 \AA$ lines seen in the IUE spectra towards HD 157042 (SWP 30914), HD 157832 (SWP 55913), HD 156575 (SWP 30888) and HD 156385 (SWP 15130), the data being obtained from the Multimission Archive at Space Telescope (MAST) (at http://archive.stsci.edu/iue/index. html). This interstellar line samples (mainly neutral) gas with an ionization potential of 8.2 to $16.3 \mathrm{eV}$ and has been shown to be a particularly good indicator of absorption features associated with the expansion of SNR's such as Shajn 147 (Phililips \& Gondhalekar 1983) and the Vela SNR (Jenkins et al. 1998). From these ultraviolet profiles we see that, as for the $\mathrm{NaI}$ lines, a marked increase in absorption equivalent width is aslo observed for the targets with distances $>1 \mathrm{kpc}$. In addition, we also observe that the velocity range over which these absorption lines are formed also shows a marked increase for the two stars (HD 156575 and HD 156385) whose distances are $>1 \mathrm{kpc}$.

Both of these stars' sight-lines lie within $1.75^{\circ}$ of each other and they are both contained within the contours of a $21 \mathrm{~cm}$ HI cavity mapped by Cappa de Nicolau et al. (1988). This HI "bubble" was mapped over the velocity range -9.6 to $+6.9 \mathrm{~km} \mathrm{~s}^{-1}$ and an expansion rate of $V \sim 10 \mathrm{~km} \mathrm{~s}^{-1}$ was derived for the neutral gas shell. In addition to the low velocity $\mathrm{NaI}$ component at $V \sim-15 \mathrm{~km} \mathrm{~s}^{-1}$ seen towards both 


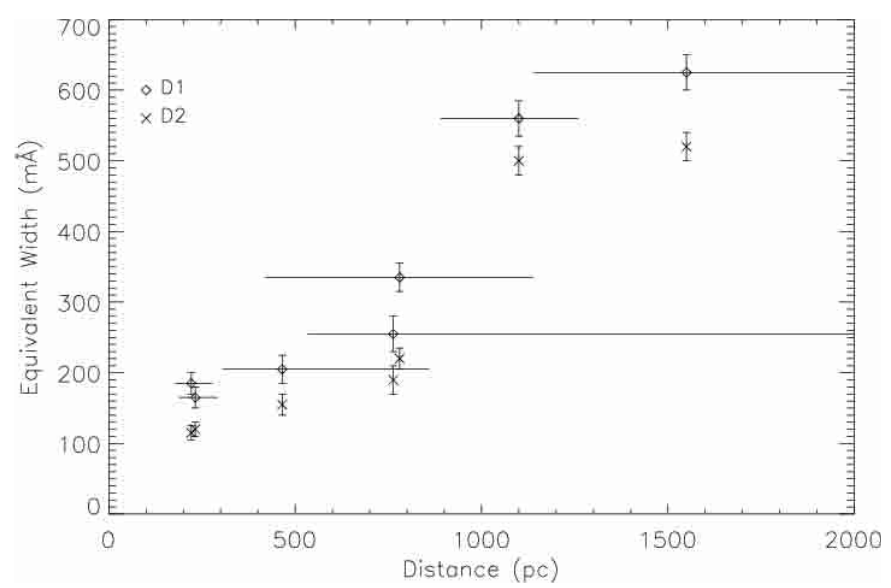

Fig. 3. Interstellar NaI D1 \& D2 equivalent widths plotted as a function of istance to the 7 observed stars. Notice the large increase in absorption values for the two most distant stars.

HD 156575 and HD 156385 (which could be due to the stellar wind-blown bubble of the WR star), we have also detected a weak absorption component at $V=-45 \mathrm{~km} \mathrm{~s}^{-1}$ in both the D2 and D1 lines observed towards HD 156385. We note that this latter component's velocity is far greater than that of the expanding HI gas shell derived by Cappa de Nicolau et al. (1988), being closer to the expansion velocity of the nebula derived from $\mathrm{H} \alpha$ emission observations (Meaburn et al. 1991), and it is of a similar magnitude to the velocity of absorption components observed towards several galactic SNRs (Welsh et al. 2001). However, Nichols \& Fesen (1994) failed to detect any isolated high-velocity $\left(V>45 \mathrm{~km} \mathrm{~s}^{-1}\right)$ absorption features in the IUE spectrum of HD 156385 that could be associated with the expansion of interstellar gas shells ("ring nebulae") that are thought to surround $\sim 30 \%$ of all WR stars (Marston 1996). Inspection of the newly processed SWP 15130 and LWR 2304 IUE spectra of HD 156385 clearly show that the resonance absorption lines of MgI $\lambda 2852 \AA$ SiII $\lambda 1304 \AA$ (see Fig. 4) and CI $\lambda 1657 \AA$ are formed over a total velocity range of $\sim 90 \mathrm{~km} \mathrm{~s}^{-1}$, consistent with both the range of absorption and the presence of an absorption component at $V=-45 \mathrm{~km} \mathrm{~s}^{-1}$ presently observed in the NaI spectra towards this star.

The coincidence of the positions of HD 156575 and HD 156383 with respect to the contours of both the radio $\mathrm{HI}$ bubble and the $\mathrm{H} \alpha$ filamentary emission from the RCW 114 nebula, taken together with the observed increase in both the complexity and velocity range of their $\mathrm{NaI}$ and SiII absorption-line spectra cannot easily be discounted as pure chance. Most WR stars are believed to have evolved from massive $\left(>25 M_{\odot}\right)$ O-type progenitors and have high massloss rates $\left(5 \times 10^{-5} M_{\odot} / \mathrm{yr}\right)$, high wind terminal velocities $\left(V_{\infty}=2000 \mathrm{~km} \mathrm{~s}^{-1}\right)$ and radiate enormous amounts of Lyman continuum photons $\left(N_{\mathrm{Lyc}} \sim 2 \times 10^{48} \mathrm{~s}^{-1}\right)$. If we assume that the distance to the $4^{\circ}$ diameter RCW 114 nebula is $\sim 1.5 \mathrm{kpc}$ (and not $200 \mathrm{pc}$ as suggested by B84), this would imply an HI bubble diameter of $\sim 100 \mathrm{pc}$. This size is quite large for a classic, single WR star wind-blown bubble, but we note that Marston (1996) has recently discovered large (concentric) IRAS shells with diameters of $\sim 100 \mathrm{pc}$ surrounding $30 \%$ of all
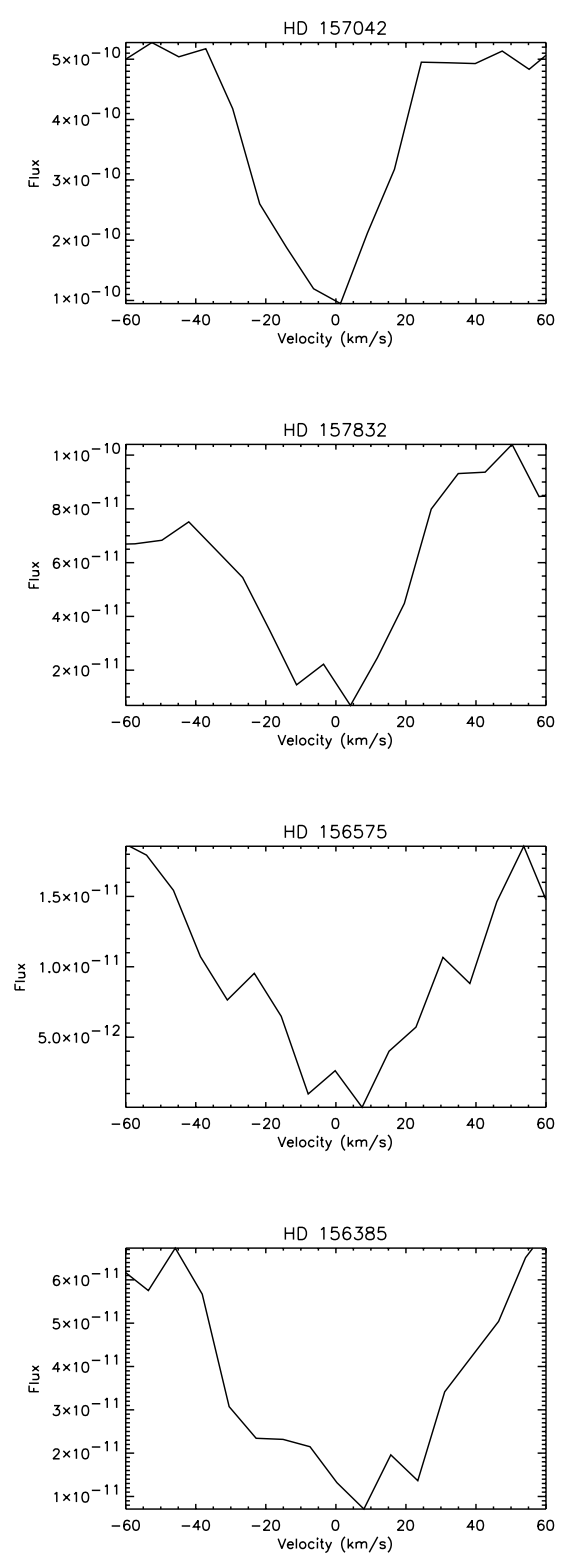

Fig. 4. IUE spectra of the SiII $\lambda 1304$ absorption line recorded towards HD $157042(d=221 \mathrm{pc})$, HD $157832(d=780 \mathrm{pc})$, HD $156575(d=$ $1100 \mathrm{pc})$ and HD $156385(d=1550 \mathrm{pc})$. Note the marked increase in absorption complexity for the two most distant stars.

galactic WR stars. These large shells mostly appear ring-like in $\mathrm{H} \alpha$ images (Marston 1995) and are not dominated by the many wispy, filamentary emission structures seen in the $\mathrm{H} \alpha$ images of the RCW 114 nebula that are so reminiscent of many galactic SNR's (Walker \& Zealey 2001). We note, however, that high-mass $\mathrm{O}$-star binaries are expected to evolve into an initial WR + O-star system, in which the WR star may eventually explode as a supernova leaving an O-star + compact companion system which will ultimately evolve into a WR + compact companion system (Vanbeveren 1991). Although only a few $\mathrm{WR}+\mathrm{O}$ star systems are presently known to exist, in the case of the RCW 114 nebula we may have the situation in which the distinctive $\mathrm{H} \alpha$ and [SII] filamentary gas emission are representative of an evolved supernova remnant (created by a progenitor WR or O-type stellar explosion) and the 
$21 \mathrm{~cm}$ emission and low velocity $\mathrm{NaI}$ absorption components are produced by the present stellar wind of the (younger) WR star, HD 156385, interacting with the ambient interstellar medium. Clearly, the future identification of a compact companion star to HD 156385 with a distance of $\sim 1.5 \mathrm{kpc}$ would support such a scenario.

Finally, we note that Marston (1995) gives the age estimate of a classic WR bubble shell, $t$, in terms of its radius, $r$, and its expansion velocity, $V_{\exp }$ as:

$t=10^{6} \times r(\mathrm{pc}) / V_{\exp }\left(\mathrm{km} \mathrm{s}^{-1}\right)$

Thus, assuming a radius of $50 \mathrm{pc}$ for the RCW 114 nebula we can derive an age of $5 \times 10^{6}$ years if an expansion velocity of $10 \mathrm{~km} \mathrm{~s}^{-1}$ is assumed, or an age of $1.1 \times 10^{6}$ years if the expansion velocity is $45 \mathrm{~km} \mathrm{~s}^{-1}$. These age estimates are very consistent with the estimated time for the progenitor O-star (in a high mass binary system) to evolve into a WR star after an initial supernova explosion (Marston 1995).

Following the work of Meaburn et al. (1991), we also note that a spherical shell of neutral gas with the same dimensions as the nebula placed at a distance of $>1 \mathrm{kpc}$ would possess a mass of $\geq 5 \times 10^{4} M_{\odot}$. Such a shell, expanding with with a velocity of $\sim 45 \mathrm{~km} \mathrm{~s}^{-1}$, would have a kinetic energy of $\geq 10^{51} \mathrm{erg}$, which is a typical value for a Type II supernova event. The fragmented and filamentary appearance of RCW 114 (see Fig. 1) suggests that the neutral gas we have presently observed is probably not all contained within such a uniform spherical shell, and thus the actual expanding nebular mass may be far less than the value given above. For example, Cappa de Nicolau et al. (1988) derive a mass of $6200 M_{\odot}$ for the swept-up HI gas of the wind-blown bubble surrounding HD 156385, which corresponds to an equivalent kinetic energy of $\sim 10^{50} \mathrm{erg}$. This energy is far greater than that which can be provided over a period of $10^{6}$ years by a typical massive O-star WR progenitor with a wind-luminosity of $1.3 \times 10^{36} \mathrm{erg} \mathrm{s}^{-1}$. Therefore, it seems likely that the pattern of emission and absorption features seen towards RCW 114 can be more plausibly explained by the ongoing interaction between the (low velocity) expansion of the WR bubble of HD 156385 with that of a pre-existing supernova remnant cavity, perhaps created by the massive proogenitor of the WR star.

\section{Conclusion}

We have obtained high $S / N$, medium resolution $\left(7 \mathrm{~km} \mathrm{~s}^{-1}\right)$ spectra of the interstellar NaI D1 and D2 lines seen towards 7 stars with distances ranging from 221 to $\sim 1550 \mathrm{pc}$ in the line-of-sight to the RCW 114 nebula. Our observed pattern of absorption features is in disagreement with the conclusions of Bedford et al. (1984) who placed a distance upper limit of $<200 \mathrm{pc}$ to this nebula. Our spectral data are consistent with a marked increase in $\mathrm{NaI}$ absorption complexity occuring only for sight-lines with distances $>1 \mathrm{kpc}$. This pattern of absorption complexity is also seen in the SiII $\lambda 1304 \AA$ lines observed towards four of the target stars. It seems likely that the WR star, HD 156385, may be responsible for the lowvelocity interstellar $\mathrm{NaI}$ absorption features observed towards
RCW 114. However, the complex nature of the $\mathrm{H} \alpha$, [SII] and [NII] emission from the filamentary gas seen in this direction is most probably linked to a pre-existing, evolved supernova remnant that could have been the progenitor of the aforementioned WR star.

Acknowledgements. We are grateful to the staff and directorate of the South African Astronomical Observatory (Cape Town). In particular, we wish to thank Dr. Luis Balona, Dr. David Buckley and Francois van Wyk for their invaluable assistance. We also thank both the referee (Prof. Meaburn) and Dr. Ken Elliott for their suggestions which have substantially improved this paper. Funding for this work was provided in part by the NASA FUSE Guest Observer Program.

\section{References}

Balona, L., \& Crampton, D. 1974, MNRAS, 166, 203

Bedford, D., Elliott, K., Ramsey, B., \& Meaburn, J. 1984, MNRAS, 210,693

Cappa de Nicolau, C., Niemela, V., Dubner, G., \& Arnal, E. 1988, AJ, 96, 1671

Dessart, L., Crowther, P., Hillier, J., et al. 2000, MNRAS, 315, 407

ESA 1997, The Hipparcos Satellite Catalogue, ESA SP-1200

Frisch, P. 1995, Space Sci. Rev., 72, 499

Gaustad, J. E., McCullough, P. R., Rosing, W., \& Van Buren, D. 2001, PASP, 113, 1326

Hanuschik, R., \& Vrancken, M. 1996, A\&A, 312, L17

Hanuschik, R., Hummel, W., Sutorius, E., Dietle, O. \& Thimm, G. 1996, A\&AS, 116, 309

Heckathorn, J., Bruhweiler, F., \& Gull, T. 1982, ApJ, 252, 230

Hobbs, L. M. 1978, ApJ, 222, 491

Jenkins, E., Wallerstein, G., \& Silk, J. 1984, ApJ, 278, 649

Jenkins, E., Tripp, T., Fitzpatrick, E., et al. 1998, ApJ, 492, L147

Kozok, J. 1985, A\&AS, 62, 7

Lallement, R., Bertin, P., Chassefiere, E., \& Scott, N. 1993, A\&A, 271, 734

Marston, A. 1995, AJ, 109, 1839

Marston, A. 1996, AJ, 112, 2828

Meaburn, J., \& Rovithis, P. 1977, ApJS, 46, L7

Meaburn, J., Goudis, C., Solomos, N., \& Laspias, V. 1991, A\&A, 252, 291

Nichols, J., \& Fesen, R. 1994, A\&A, 291, 283

Penprase, B. 1993, ApJS, 88, 433

Phillips, A., \& Godhalekar, P. 1983, MNRAS, 202, 483

Prinja, R. 1989, MNRAS, 241, 721

Savage, B. D., Massa, D., Meade, M., \& Wesselius, P. R. 1985, ApJS, 59,397

Sfeir, D. 1999, Ph.D. Thesis, University of Paris 6

Sfeir, D., Lallement, R., Crifo, F., \& Welsh, B. Y. 1999, A\&A, 346, 785

Vanbeveren, D. 1991, A\&A, 252, 159

Walker, A., \& Zealey, W. 2001, MNRAS, 325, 287

Welsh, B. Y., Sasseen, T., Craig, N., Jelinsky, S., \& Albert, C. 1997, ApJS, 112, 507

Welsh, B. Y., Sfeir, D., Sallmen, S., \& Lallement, R. 2001, A\&A, 372, 516

Welsh, B. Y., Sallmen, S., Sfeir, D., \& Lallement, R. 2002, A\&A, 391, 705 